\title{
Study On The Algorithm Of Multifunctional Bone External Fixator
}

\author{
Chen Jianwen ${ }^{1}$, Shi Xuewei ${ }^{2}$, Wei Mengting ${ }^{2}$ and Sun $\mathrm{Hao}^{2, \mathrm{a}}$ \\ 1 National Research Center for Rehabilitation Technical Aids, Beijing, 100176, China \\ 2 School of Control Engineering, Hebei University of Technology, Tianjin, 300130, China
}

\begin{abstract}
This paper discussed the problem that parameters of bone external fixator are difficult to calculate in the practical application. Positive solution is described in detail. We used MATLAB software to make simulation experiment. The innovation lies the development idea of using inverse position to verify the accuracy of positive solution. Forward displacement analysis was mainly developed using analytical method, which has many advantages, such as small dependence to the inverse solution and the higher precision. With further software development, we will have the algorithms; research model and interface program connected and form high precision smart compliance multifunctional bone external fixator products, which will greatly enhance the overall level of bone external fixation technology and clinical application treatment.
\end{abstract}

Keywords: bone external fixator, parallel mechanism, positive solution, inverse solution.

\section{Introduction}

In order to increase the level of clinical application of the bone external fixator, this title discuss the problem of the solution in calculating parameters in the background of related software of bone external fixator which has self-property right.

Bone external fixator uses the Taylor structure in practical application. It has six flexible studdles that connected to the stationary hoop. And it can rotate freely in the joint. When adjusting the length of the studdle, the relative location of the two hoops changes. This Taylor structure can regard as Stewart platform[1-2]. Therefore, this study can regard as the inverse solution and positive solution of the Stewart platform[3-5].Fig.1 shows the application of Stewart platform in femur correction.
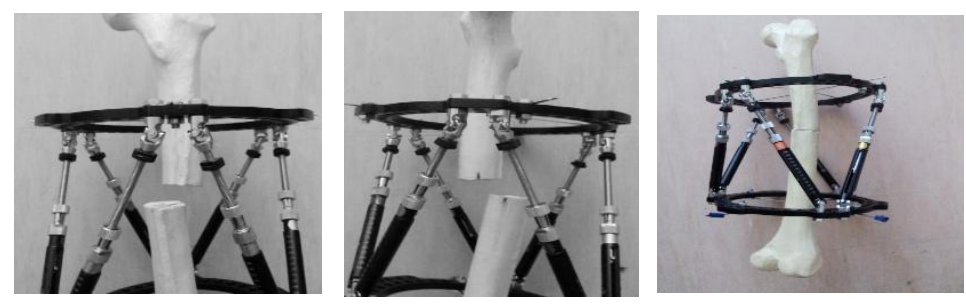

Fig 1. The application of Taylor

\footnotetext{
${ }^{a}$ Corresponding author: sunhao@hebut.edu.cn
} 


\section{Modeling}

In order to describe Stewart platform which has six axis, we must build its mathematical model. Firstly, build two coordinate systems: the base coordinate system $\{B\}$ (the origin is in the ob which is the center of the base)and the upper platform coordinate system $\{\mathrm{P}\}$ (the origin is in the op which is the center of the platform),as shown in Fig.2.

In the upper platform coordinate system , the ZP axis point to the platform's upside, the YP axis and the connecting line of $\mathrm{p} 3$ and $\mathrm{p} 4$ are vertical. The angle of p4 and p5 is defined as $\theta_{p}=15^{\circ}$, and the other points of junction distribute symmetrically. The angle of $\mathrm{p} 1$ and $\mathrm{p} 3$ is equal to the angle of $\mathrm{p} 3$ and $\mathrm{p} 5$, It is $120^{\circ}$,as shown in Fig.2 and Fig.3.In the base coordinate system ,the $\mathrm{Zb}$ axis point to the stationary platform's upside, the $\mathrm{Yb}$ axis and the connecting line of $\mathrm{B} 3$ and B4 are vertical. The angle of B3 and B4 is defined as $\theta_{b}=15^{\circ}$, and the other points of junction distribute symmetrically. The angle of B1 and B3 is equal to the angle of B4 and B6,It is $120^{\circ}$,as shown in Fig.4.

Secondly, define the angle of the Pi axis and the XP axis as $\lambda_{\mathrm{i}}$ and define the angle of the Bi axis and the $\mathrm{Xb}$ axis as $\Lambda_{i},(\mathrm{i}=1,2, \ldots, 6)$.we can get some relationship:

$$
\begin{array}{ll}
\Lambda_{1}=330^{\circ}-\frac{\theta_{b}}{2}, & \Lambda_{2}=330^{\circ}+\frac{\theta_{b}}{2}, \\
\Lambda_{3}=90^{\circ}-\frac{\theta_{b}}{2}, & \Lambda_{4}=90 \frac{\theta_{b}}{2}, \\
\Lambda_{5}=210^{\circ}-\frac{\theta_{b}}{2}, & \Lambda_{6}=210^{\circ}+\frac{\theta_{b}}{2}, \\
\lambda_{1}=270^{\circ}+\frac{\theta_{p}}{2}, & \lambda_{2}=30^{\circ}-\frac{\theta_{p}}{2}, \\
\lambda_{3}=30^{\circ}+\frac{\theta_{p}}{2}, & \lambda_{4}=150^{\circ}-\frac{\theta_{p}}{2}, \\
\lambda_{5}=150^{\circ}+\frac{\theta_{p}}{2}, & \lambda_{6}=270^{\circ}-\frac{\theta_{p}}{2} .
\end{array}
$$

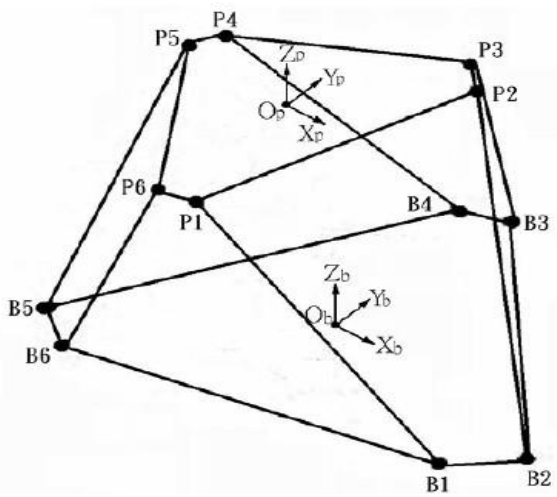

Fig 2.The define of the coordinate.

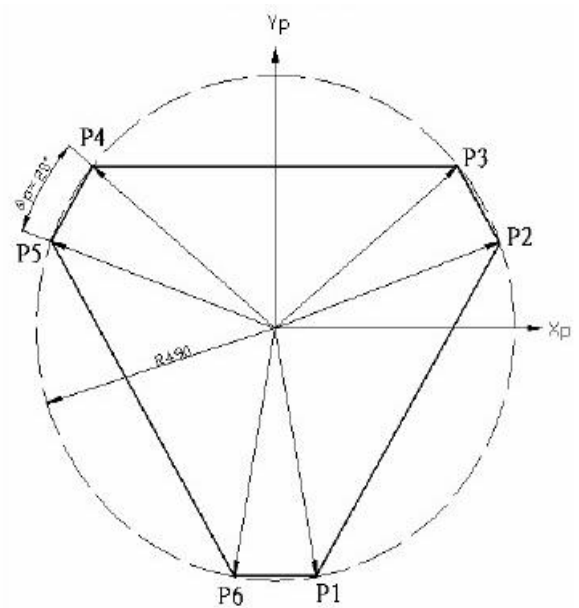

Fig 3. The joint angle of the upper.

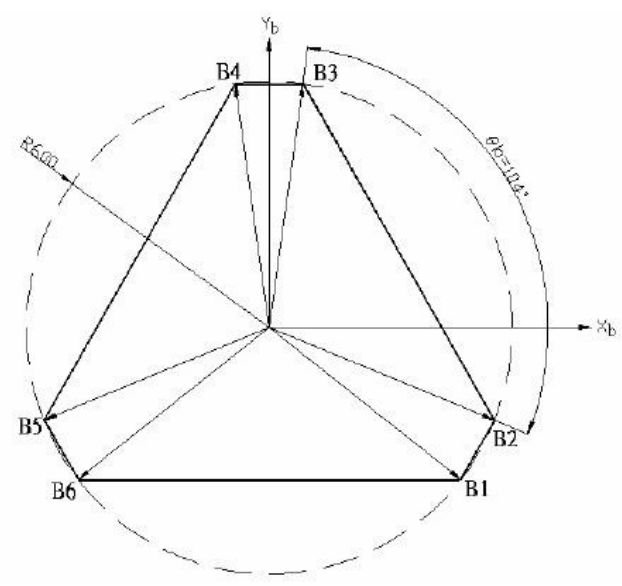

Fig 4. The joint angle of the lower.

Thirdly, define the vector of the point $P_{i}$ which is in the $\{\mathrm{P}\}$ system as ${ }^{P} p_{i}=\left(\mathrm{p}_{i x} \mathrm{p}_{i y} \mathrm{p}_{i z}\right)^{T}$ and define 
the vector of the point $B_{i}$ which is in the $\{\mathrm{B}\}$

system as ${ }^{B} b_{i}=\left(\begin{array}{lll}\mathrm{b}_{i x} & \mathrm{~b}_{i y} & \mathrm{~b}_{i z}\end{array}\right)^{T}$.Then:

$$
\begin{aligned}
& { }^{P} p_{i}=\left[\begin{array}{lll}
\mathrm{r}_{p} \cos \left(\lambda_{i}\right) & \mathrm{r}_{p} \sin \left(\lambda_{i}\right) & 0
\end{array}\right]^{T} \\
& { }^{B} b_{i}=\left[\begin{array}{lll}
\mathrm{r}_{b} \cos \left(\Lambda_{i}\right) & \mathrm{r}_{b} \sin \left(\Lambda_{i}\right) & 0
\end{array}\right]^{T}
\end{aligned}
$$

$r_{p}$ is the platforms circumradius and $r_{b}$ is the base's circumradius. The last, we define two working spaces: Cartesian Space: $\left[\begin{array}{llllll}\mathrm{x} & \mathrm{y} & \mathrm{z} & \alpha & \beta & \gamma\end{array}\right]^{T} \quad ; \quad$ Joint $\quad$ Space: $\left[\begin{array}{llllll}\mathrm{S}_{1} & \mathrm{~S}_{2} & \mathrm{~S}_{3} & \mathrm{~S}_{4} & \mathrm{~S}_{5} & \mathrm{~S}_{6}\end{array}\right]^{T}$

$\left[\begin{array}{lll}\mathrm{x} & \mathrm{y} & \mathrm{z}\end{array}\right]^{T}$ is the location vector of the upper platform; $\left[\begin{array}{lll}\mathrm{x} & \mathrm{y} & \mathrm{z}\end{array}\right]^{T}$ is the attitude angle vector of the upper platform; $\left[\mathrm{S}_{1} \mathrm{~S}_{2} \mathrm{~S}_{3} \mathrm{~S}_{4} \mathrm{~S}_{5} \mathrm{~S}_{6}\right]^{T}$ is the length vector of the six studdles. Till then, the mathematical model of the parallel structure is done.

\section{Positive solution algorithm}

When knows the six inputs, we just use the analytical method to solve. Here we establish the upper platform's coordinate system: $O-X Y Z$ and establish the lower platforms coordinate system: $O-X Y Z$. They have the relationship:

$$
X=[T] X^{\prime}+P
$$

Among them:

$$
\begin{gathered}
{[T]=\left[\begin{array}{lll}
d_{11} & d_{12} & d_{13} \\
d_{21} & d_{22} & d_{23} \\
d_{31} & d_{32} & d_{33}
\end{array}\right],} \\
P=\left\{\begin{array}{lll}
X_{P} & Y_{P} & Z_{P}
\end{array}\right\}^{T}
\end{gathered}
$$

In the eq.(3), $|T|$ is the direction cosine matrix of the upper; $P$ is the position vector of the upper's center in the fixed-coordinate system. With the spatial geometric relationship of the parallel mechanism, when know the sizes, we can solve the coordinate value of each hinge point of the moving platform in the $O-X Y Z$ system. Then the vector of the driver's length in the fixed-coordinate system is:

$$
l_{i}=\left(b_{i}-B_{i}\right), \quad i=1,2 \ldots
$$

The equation of the inverse solution of the parallel mechanism is as follows:

$$
l_{i}=\sqrt{l_{i x}^{2}+l_{i y}^{2}+l_{i z}^{2}}, \quad i=1,2 \ldots \ldots 6
$$

Divide the six independent equations in eqn.(5) into three part: $\left(\begin{array}{ll}l_{1}^{1} & l_{6}^{2}\end{array}\right),\left(\begin{array}{ll}l_{2}^{2} & l_{5}^{2}\end{array}\right)$ and $\left(\begin{array}{ll}l_{3}^{2} & l_{4}^{2}\end{array}\right)$, and do the addition and subtraction about them, and simplify, we get six independent equations in other form. And by using some constraint equations and relationships we can get :

$$
\begin{aligned}
F_{1}= & \left(l_{3}^{2}+l_{4}^{2}\right) / 2-\left(R^{2}+R_{0}^{2}\right)-W \\
& -2 Y_{p} B_{3 Y}+2\left(d_{11} b_{3 X}^{\prime} B_{3 X}+d_{22} b_{3 Y}^{\prime} B_{3 Y}\right) \\
& -2 b_{3 Y}^{\prime}\left(d_{12} X_{P}+d_{22} Y_{P}+d_{32} Z_{P}\right)=0 \\
F_{2}= & \left(l_{3}^{2}-l_{4}^{2}\right) / 4+X_{P} B_{2 X}+d_{21} b_{3 X}^{\prime} B_{3 Y}+ \\
& d_{12} b_{3 X}^{\prime} B_{3 Y}-b_{3 X}^{\prime}\left(d_{11} X_{P}+d_{21} Y_{P}+d_{31} Z_{P}\right) \\
& =0 \\
F_{3} & =d_{11} d_{12}+d_{21} d_{22}+d_{31} d_{32}=0
\end{aligned}
$$

Solve the equations with the optimization theory. Its objective function is composed by least square principle.

It is:

$$
S(X Y Z)=\sum_{j=1}^{3} F_{i}^{2}(X, Y, Z)
$$

\section{Simulation result}

\footnotetext{
a Corresponding author: sunhao@hebut.edu.cn
} 
As shown in Table 1, the relative error is small.

It can reach the level of $10^{-2}$. The iterative calculation lead to the relative error. It is within the acceptable level.

Table 1.Experimental data

\begin{tabular}{|c|c|c|c|}
\hline No & $\begin{array}{c}\text { Inputs } \\
\text { ( length) }\end{array}$ & $\begin{array}{c}\text { Positive } \\
\text { Outputs } \\
\text { (pose) }\end{array}$ & $\begin{array}{c}\text { Relative } \\
\text { error }\end{array}$ \\
\hline 1 & {$\left[\begin{array}{l}150 \\
167 \\
155 \\
165 \\
157 \\
160\end{array}\right]$} & {$\left[\begin{array}{c}9.11 \\
4.63 \\
105.68 \\
-0.0978 \\
-0.0049 \\
-0.0534\end{array}\right]$} & $\begin{array}{c}0.01 \% \\
-0.20 \% \\
-0.21 \% \\
0.14 \% \\
0.20 \% \\
0.06 \%\end{array}$ \\
\hline 2 & {$\left[\begin{array}{l}124 \\
112 \\
103 \\
126 \\
123 \\
137\end{array}\right]$} & {$\left[\begin{array}{c}2.36 \\
0.3 \\
105.06 \\
0.1096 \\
0.1124 \\
-0.1396\end{array}\right]$} & $\begin{array}{c}0.18 \% \\
2.10 \% \\
2.69 \% \\
-1.44 \% \\
-2.17 \% \\
-0.64 \%\end{array}$ \\
\hline 3 & {$\left[\begin{array}{l}127 \\
116 \\
120 \\
124 \\
127 \\
113\end{array}\right]$} & {$\left[\begin{array}{c}-8.78 \\
-0.2 \\
105.21 \\
-0.0989 \\
-0.0989 \\
-0.0209\end{array}\right.$} & {$\left[\begin{array}{c}3.14 \% \\
6.38 \% \\
4.23 \% \\
-7.32 \% \\
-7.11 \% \\
1.72 \%\end{array}\right]$} \\
\hline 4 & {$\left[\begin{array}{ll}1 & 17 \\
1 & 16 \\
122 \\
130 \\
116 \\
129\end{array}\right]$} & {$\left[\begin{array}{c}5.24 \\
-13.14 \\
105.23 \\
0.0983 \\
0.0408 \\
-0.0291\end{array}\right]$} & {$\left[\begin{array}{c}0.69 \% \\
4.40 \% \\
-4.68 \% \\
-4.26 \% \\
4.15 \% \\
0.69 \%\end{array}\right]$} \\
\hline 5 & $\left.\begin{array}{c}129 \\
144 \\
125 \\
75 \\
116 \\
117\end{array}\right]$ & {$\left[\begin{array}{c}4.9389 \\
30.7393 \\
96.9103 \\
-0.0738 \\
-0.3654 \\
-0.1884\end{array}\right.$} & {$\left[\begin{array}{c}-0.91 \% \\
0.38 \% \\
0.23 \% \\
0.97 \% \\
-0.02 \% \\
-0.50 \%\end{array}\right]$} \\
\hline
\end{tabular}

\section{Summary}

At the present stage, the technology of bone fixing in the external has been the important part in the field of bone's cure. This study is working on solving the problem that the parameters of bone external fixator are difficult to calculate in the practical application.

In the study of the Stewart platform, the analysis of positive solution is the basement of its application. The paper study mainly study the forward displacement analysis used analytical method that with many advantages, such as programming faster, calculate easier, higher precision, especially the initial value calculatio-n is more easier to get. We can get the pose of the upper platform through inputting the length of six bars. By this way we can make the bones rest orate gradually. And this method supply a sufficient way about the application of positive solution.

\section{Acknowledgements}

The research work was supported by Key Projects in the National Science \& Technology Pillar Program during the 12th Five-year Plan Period of China under Grant No. 2012BAI33B06.

\section{References}

1. Huang Zhen, Zhao Yongsheng, and Zhao Tieshi. Higher Spatial Kinematics. Beijing:Higher Education Press.pp.141-158,Jun.2006.

2. Huang Zhen, Kong Lingfu and Fang Yuefa.The Parallel Robot Manipulator and Its Mechanism Theory and Control.China Machine Press.pp.1-346,1997.

3. Gao Hong, and Zhao Han. Areview about theory of mechanism for parallel robotics[J].Journal of Anhui University of Technology and Science, 21,pp.73-79, 2006.

4. Wang Xuesong, Hao Minglin, and Cheng Yuhu,et al. Forward Kinematics of Parallel Manipulators Based on Differential Evolution[J].Journal of China University of Mining\&Technology, 37,pp.664-669, 2008.

5. Liu Huilin, Zhang Tongzhuang, and Ding Hongsheng. Forward Solutions of the 
ICMEE 2015

3-RPR Planar Parallel Mechanism with Wu's Method[J].Journal of Beijing Institute of Technology, 42,pp.229-233,2011. 